\title{
Dorsal Medial Prefrontal Cortex Plays a Necessary Role in Rapid Error Prediction in Humans
}

\author{
Mandana Modirrousta and Lesley K. Fellows \\ Department of Neurology and Neurosurgery, McGill University, Montreal Neurological Institute, Montreal, Quebec, Canada H3A 2B4
}

Activity in human dorsal anterior cingulate cortex (dACC) is correlated with errors, near-misses, and response conflict. Based on these observations, this region has been cast as playing a central role in models of error processing, conflict monitoring, and cognitive control. However, clear evidence that this region of the brain is necessary for these processes has been elusive. We studied the effects of damage to this region on four different error-related measures in five patients, and 19 healthy participants. Most error-related indices were not affected by such damage: patients had intact post-error slowing, and were able to report and to correct errors after they were made with accuracies comparable with the control group. However, all five patients were notably slow to correct errors, suggesting a deficit in on-line error prediction. This slowing was associated with impairment in the conscious prediction of error likelihood before a response. This finding constitutes important converging evidence for a critical role for human dACC in error monitoring, and sheds light on the selectivity and timing of the error-related process affected by dACC damage.

Key words: anterior cingulate cortex; conflict monitoring; cognitive control; executive function; decision making; lesion

\section{Introduction}

In a setting where action is required, the adaptive solution is to produce the optimal response at the minimum cost. When the environment suggests more than one appropriate response and calls for a fast decision, the challenge is to respond both quickly and accurately: too slow, and the opportunity may be lost; too fast, and the wrong response may be selected; too much equivocation, and no response will be produced at all. The neural systems engaged to solve this problem in the human brain have been studied extensively using a variety of methods. Functional imaging studies have shown that dorsal anterior cingulate cortex (dACC) is more active in diverse tasks that involve choosing between incompatible responses (Ridderinkhof et al., 2004; Carter and van Veen, 2007; Rushworth et al., 2007). Electrophysiological studies have identified response conflict-related waveforms that likely arise from the same region (Yeung et al., 2004), and that relate to performance adjustment on subsequent trials (Gehring et al., 1993; Debener et al., 2005). Similar activity has been identified with both methods in response to errors, which can be considered as response conflict taken to its extreme (Gehring et al., 1993; Carter et al., 1998; Ridderinkhof et al., 2004).

Although the specific processes supported by dACC remain a

Received Sept. 16, 2008; revised 0ct. 31, 2008; accepted Nov. 16, 2008.

This work was supported by Canadian Institutes of Health Research (CIHR) Grant MOP-77583 and National Institutes of Health-National Institute of Neurological Disorders and Stroke Grant NS045074. L.K.F. is a CIHR Clinician-Scientist and Montreal Neurological Institute Killam Scholar. We thank the database managers at both the University of Pennsylvania (Marianna Stark) and McGill University (Arlene Berg) for their help with patient recruitment, and the patients and their families for their generous participation. Alisa Padon provided expert technical assistance. Matt Botvinick and Brenda Milner made helpful comments on earlier drafts of this manuscript.

Correspondence should be addressed to Lesley K. Fellows, Department of Neurology and Neurosurgery, McGill University, Montreal Neurological Institute, 3801 University Street, Montreal, Quebec, Canada H3A 2B4. E-mail: lesley.fellows@mcgill.ca.

DOI:10.1523/JNEUROSCI.4450-08.2008

Copyright $\odot 2008$ Society for Neuroscience $\quad$ 0270-6474/08/2814000-06\$15.00/0 matter of debate, this body of work certainly suggests a central role for dACC in cognitive control. It is thus remarkable that it has been so difficult to show unequivocally that this region is, in fact, necessary for conflict monitoring or cognitive control in humans (Swick and Turken, 2002; Fellows and Farah, 2005; Naccache et al., 2005; Baird et al., 2006; Floden and Stuss, 2006; di Pellegrino et al., 2007; Picton et al., 2007). While response conflict is computationally well defined, debate continues as to its behavioral hallmarks (Mayr et al., 2003; Ullsperger et al., 2005; Eichele et al., 2008; Verguts and Notebaert, 2008). The lack of a "gold standard" behavioral measure, in addition to the rarity of focal damage to dACC, may go some way to explaining the conflicting neuropsychological literature to date. Here, we focused on the potentially simpler phenomenon of outright response errors. We aimed to determine which, if any, components of error processing are affected by dACC damage in humans. Five patients with focal damage affecting dorsal mPFC, centered on dACC, performed versions of a speeded choice reaction time task, the Eriksen flanker task, designed to permit measurement of error correction, error reporting, and post-error slowing. Such damage had subtle, but consistent effects only on the timing of error correction, suggesting a selective deficit in the within-trial anticipation of an error. This difficulty with error prediction was confirmed in a second task, a modified n-back working memory task which probed participants' overt confidence in their responses at the time the responses were made. DACC damage was associated with a misplaced confidence in responses that were, in fact, errors. These findings argue that intact dACC is necessary for the rapid, on-line anticipation of errors.

\section{Materials and Methods}

Subjects. Subjects with medial prefrontal cortex (mPFC) damage encompassing dACC were identified through the McGill Cognitive Neuro- 
science Research Registry, and the database of the Center for Cognitive Neuroscience at the University of Pennsylvania. Lesions were secondary to ischemic stroke in either the anterior cerebral artery or peri-callosal artery territory, and had occurred at least 6 months before testing (range 6 months-11 years). Individual lesions were traced from the most recent clinical magnetic resonance imaging onto the standard Montreal Neurological Institute brain by a neurologist with experience in image analysis and blind to the behavioral data, using MRIcro software (Rorden and Brett, 2000). Three patients had lesions limited to the left hemisphere, centered in pre-genual and dorsal ACC and two patients had bilateral lesions which involved bilateral mPFC, including dACC, and extending into bilateral frontopolar and orbitofrontal cortex.

Age- and education-matched control subjects were drawn from a database of healthy volunteers recruited from the local Montreal community by advertisement. Control participants (CTL; $n=19$ ) were free of neurologic or psychiatric disease, substance abuse, or closed head injury. None were taking psychoactive medication, and all scored at least 27/30 on the Montreal Cognitive Assessment (http://www.mocatest.org/). All participants provided written informed consent, in accordance with the principles set out in the Declaration of Helsinki and the stipulations of the local Institutional Review Boards, and received a nominal fee as compensation for their time and effort.

Tasks. Participants completed three versions of the Eriksen flanker task, each consisting of 400 trials, to measure post-error slowing, error correction, and error reporting, respectively. A fourth version that included a perceptual detection task was included as a control for any dual task demands of the error detection and reporting versions. All participants performed the tasks in the same order. In all versions, subjects viewed a series of 5 arrows on a laptop computer screen (within a rectangular fixation frame that remained on the screen), and were instructed to indicate the direction of the central arrow by pressing the left or right response key with the index and middle fingers of their preferred hand as quickly and accurately as possible. An equal number of congruent $(\ll \ll$ or $\ggg \gg)$ and incongruent $(\ll>\ll$ or $\gg<\gg)$ stimuli were presented, randomly intermixed, in each block of 200 trials. A feedback screen occurred every 20 trials, providing the mean reaction time (RT) for those trials, accompanied by written instructions to continue to respond as quickly and accurately as possible. No explicit feedback was given about accuracy.

The first, "standard," version required only a single response. The measure of interest was post-error slowing, i.e., the mean RT of correct trials that followed error trials, compared with correct trials that followed correct trials. The second, "error reporting," version required participants to push a separate key (with their other hand) on trials in which they believed they had made an error. The third, "error-correction," version had subjects make a second, corrective response by pushing the alternate response key as quickly as possible, if they believed their first response was an error. Ten practice trials were provided at the beginning of each task, and could be repeated if the participant so desired.

Because the focus of these experiments was on error-related processes, and in keeping with at least one recent study (Vocat et al., 2008), task parameters were adjusted to ensure approximately comparable error rates within the mPFC group, and between $\mathrm{mPFC}$ and control groups. Stimulus presentation times and interstimulus intervals (ISIs) thus differed in the two groups. Controls viewed each stimulus for $100 \mathrm{~ms}$, and then had $1000 \mathrm{~ms}$ in which to respond. This was followed by a blank screen for $200 \mathrm{~ms}$. An additional $500 \mathrm{~ms}$ was added to the response window in the "error detection" blocks, and $1000 \mathrm{~ms}$ in the error reporting blocks, to allow adequate time to record the second response, if appropriate. These parameters resulted in an error rate of $\sim 8 \%$, on average.

All five patients required adjustments in task timing to perform at levels of accuracy that were comparable with controls. Stimuli were presented for $250 \mathrm{~ms}$, and the ISI was increased to $800 \mathrm{~ms}$. The response window was adjusted for each individual, based on their performance of a simple RT task (completed as part of a separate experiment, on a prior occasion), and in a few cases, further adjusted after the first block of the standard flanker task. Response deadlines varied between 1000 and 2000 $\mathrm{ms}$ across the three versions of the task. One patient, who had suffered extensive bilateral medial frontal, frontopolar, and orbitofrontal damage, had difficulty following the instructions for the flanker tasks, required extra practice to learn both the standard and error correction version of the task, and was unable to carry out the error reporting version, despite additional practice.

We wanted to ensure that any deficits in error monitoring did not reflect general difficulties with dual task performance, and so included a control version of the standard Eriksen flanker task paired with a second, "perceptual reporting" task. In this control task, subjects performed the flanker task, but were instructed to press a separate key, immediately after the flanker response, if they had detected that the frame around the arrows had blinked during the trial. The duration of stimulus presentation, the ISI, and the response deadlines in this task were identical to those of the error reporting task for controls and for each patient. The frame blinked for $15 \mathrm{~ms}$ in a randomly selected $20 \%$ of trials, with the blink occurring within a $350-450 \mathrm{~ms}$ time window after the onset of the arrow display.

In a second experiment, all five patients and 10 of the control participants performed a modified version of a letter two-back task that provided an explicit measure of on-line error likelihood at the time of the response. Subjects viewed a series of letters, presented one at a time, and pressed one of two keys each time the letter shown was the same as the letter shown 2 trials before. They were instructed to press one key to indicate that they were "certain" the stimulus was a target and a second key if they were "somewhat certain." If they thought the stimulus was not a target, they made no response. There were 20 targets in a total of 120 trials, so most trials did not require a response. Our previous work with a standard, single response form of this 2-back task had shown that patients with $\mathrm{mPFC}$ damage were more likely to make errors of commission (Tsuchida and Fellows, 2008). This variant allowed us to measure whether they were able to estimate the likelihood that a given response was an error at the time of the response.

\section{Results}

Figure 1 shows lesion extent and overlap for all five patients. Damage was bilateral in two patients, and affected only left dorsal $\mathrm{mPFC}$ in 3 . The common area damaged in all 5 subjects included dorsal and pre-genual ACC. Nineteen age- and educationmatched healthy control subjects were recruited from the community. Demographic information is summarized in Table 1. There was no significant difference between the groups with regard to age $(t=-0.07 ; p>0.05)$, education $(t=-1.25, p>$ $0.05)$, or IQ estimated by the American version of the National Adult Reading Test (ANART) $(t=1.65, p>0.05)$.

Subjects completed three versions of the Eriksen flanker task, each probing a (potentially) different aspect of error processing. No explicit accuracy feedback was provided during any of these versions. Because the focus was on error-related processes, task difficulty was adjusted by varying stimulus presentation and response windows so that patients and controls had comparable error rates $(\sim 8 \%$ in the standard task). In the standard version of the task, patients were on average slower, and showed a larger flanker effect (i.e., slowing was more marked for incongruent than congruent trials) (Table 2). These effects varied across patients, however. Only 2 of 5 showed slowing on incongruent trials that was $>1.5$ SD from the control mean, and only 3 of 5 had flanker effects that were $>1.5 \mathrm{SD}$ from the control mean, arguing that flanker performance per se is not necessarily disrupted by mPFC damage.

We first examined the effect of an error on performance on the subsequent trial: the mPFC group had intact post-error slowing, of similar magnitude to the post-error slowing seen in the control group (ANOVA; effect of trial type $F_{(1,22)}=8.0, p=0.01$; no significant group $\times$ trial type interaction). The mean (SD) RT of correct trials following errors was 640 (186) ms, and of correct 


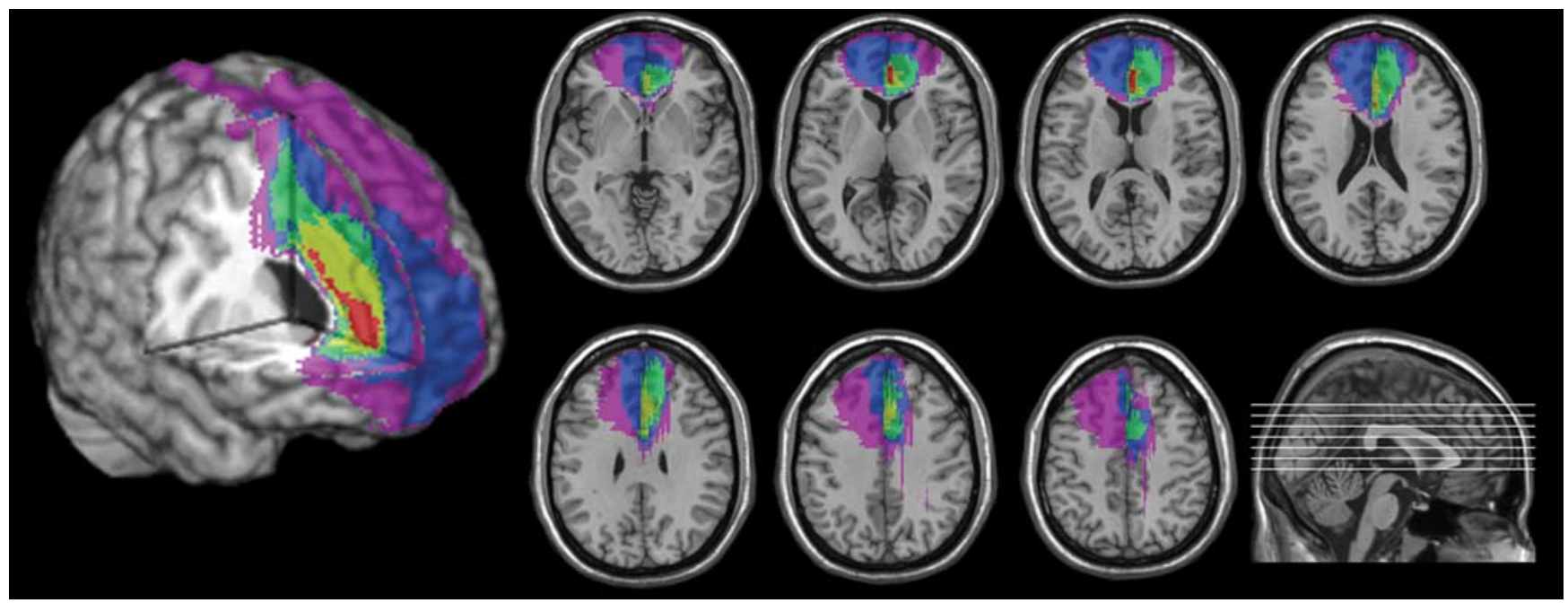

Figure 1. Lesion location and overlap for the $\mathrm{mPFC}$ group, shown on axial slices of the standard Montreal Neurological Institute brain oriented according to radiological convention (i.e., left is right), and on a three-dimensional reconstruction cut away to show the left medial frontal lobe. Pink indicates regions damaged in one subject; blue, in two subjects; green, in three subjects; yellow, in four subjects; and red, the common area affected in all five subjects.

Table 1. Demographic information [mean (SD)]

\begin{tabular}{lllll}
\hline Group & Age & Education (years) & Sex (F/M) & ANART IQ estimate \\
\hline $\operatorname{CTL}(n=19)$ & $58.74(12.6)$ & $12.32(2.4)$ & $12 / 7$ & $122.4(7.7)$ \\
$\operatorname{mPFC}(n=5)$ & $59.2(15.1)$ & $13.8(2.2)$ & $2 / 3$ & $116.2(5.4)$ \\
\hline
\end{tabular}

$\mathrm{F}$, Female; $\mathrm{M}$, male.

Table 2. Flanker task performance

\begin{tabular}{lllll}
\hline Group & RT (c) (ms) & RT (ic) (ms) & Errors (c) (\%) & Errors (\%) \\
\hline CTL $(n=19)$ & $454.2(67.8)$ & $480.1(74.9)$ & $2.9(4.7)$ & $5.3(4.6)$ \\
mPFC $(n=5)$ & $542.1(152.6)$ & $649.6(257.5)$ & $1.4(1.2)$ & $6.7(2.5)$ \\
\hline
\end{tabular}

c, Congruent trials; ic, incongruent trials.

trials following correct trials was 586 (194) $\mathrm{ms}$ in the mPFC group [CTL post-error RT: 490 (94) ms, post-correct RT: 466 (69) ms].

In a second version of the flanker task, participants were required to report that an error had been made by pressing a separate key with the other hand after error commission. One patient with extensive damage beyond dorsal $\mathrm{mPFC}$ was unable to perform this version of the task, a difficulty that seemed to be related to problems understanding the instructions. In the other four patients, dorsal mPFC damage did not significantly interfere with the ability to report errors in this way: both groups reported a similar proportion of error trials as errors [mPFC: 43 (24) \%; CTL: $\left.64(25) ; t_{(21)}=1.49, p>0.05\right]$, and did so with a similar RT [mPFC: 776 (279), CTL: $627(153) ; t_{(21)}=-1.7, p>0.05$ ]. These findings are summarized in Figure 2. Seven subjects in the control group and two patients also reported at least one correct response as an error; the frequency of such "false error reports" did not differ between groups (Fisher's exact test, $p>0.05$ ).

A third version of the flanker task required participants to correct any errors, by pressing the alternate response key as quickly as possible. The mPFC group (including all five patients) and the CTL group were again equally able to recognize errors after they had been made, correcting [mean (SD)] 66 (36) \% and $68(28) \%$ of error trials, respectively $\left(t_{(22)}=0.14, p>0.05\right)$. Prior work has shown that such error correction is relatively rapid, typically occurring in 200-250 ms (Rabbitt, 1966). The control group confirmed this pattern, correcting errors 223 (78) $\mathrm{ms}$ after the error response, on average. Despite the normal rate of error

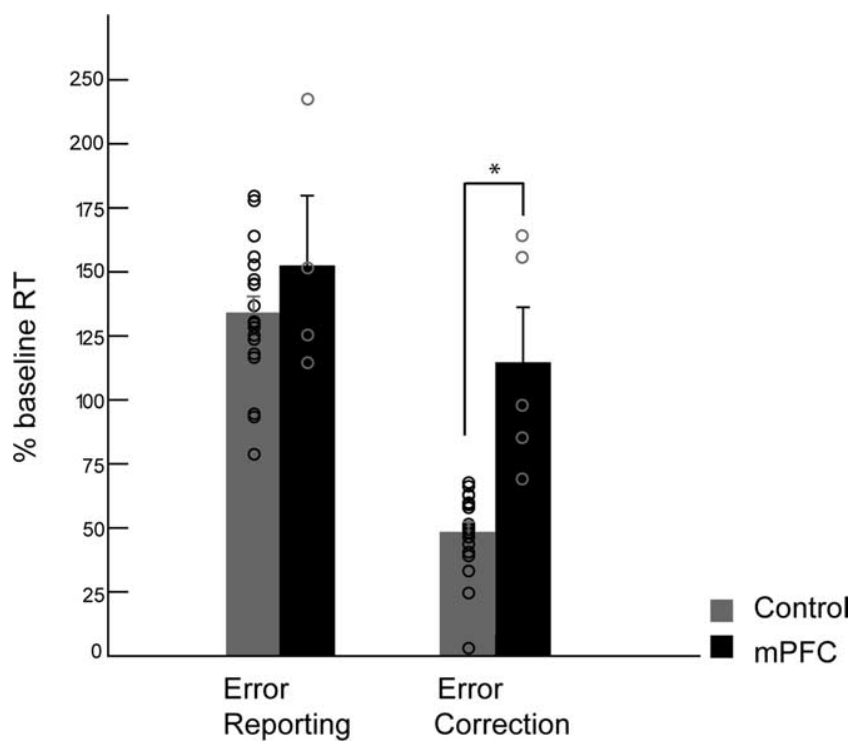

Figure 2. Reaction times for error reporting and error correction in the Eriksen flanker task. Response times were, in general, slower for the mPFC group. The time to report and correct errors is shown here normalized to the choice RT in the standard Eriksen flanker task for each subject. Statistical effects were similar regardless of whether normalized or absolute RTs were tested. The asterisk indicates significantly slower error correction RT in those with mPFC damage compared to controls, $p<0.05$.

correction in the mPFC group, these patients were notably slow to make the corrective response: mean RT 709 (458) ms (significantly slower than CTL; $t_{(22)}=-4.65, p<0.001$ ) (Fig. 2). Importantly, the slowing of error correction RT was evident in the individual performance of all five mPFC patients: the fastest patient had an error correction RT that was 1.43 SD from the control mean, and the remaining four were $>1.9$ SD from the mean. This is not an artifact of overall slowing in these patients. The same pattern holds whether the results are analyzed in absolute terms, or expressed as a proportion of each individual's RT on the standard version of the flanker task. The within-subject analysis (limited to the four patients who completed both tasks) confirms this finding: there are significant main effects of group $\left(F_{(1,21)}=\right.$ $9.7, p<0.01)$ and of $\operatorname{task}\left(F_{(1,21)}=73, p<0.001\right)$, indicating that 
the patient group was overall slower, and that error correction was faster than error reporting, as expected. Critically, the interaction between task and group is also significant $\left(F_{(1,21)}=5.2\right.$, $p<0.05)$, reflecting the disproportionate slowing of error correction compared with error reporting in those with $\mathrm{mPFC}$ damage.

A priori, we considered the possibility that impaired error reporting or correction might reflect a nonspecific difficulty performing what could be considered a dual task. We therefore included a control task that combined the flanker task with a requirement to report a brief perceptual event (a $15 \mathrm{~ms}$ blink of the fixation frame occurring 350-450 ms after the onset of the flanker arrow stimuli, on $20 \%$ of trials), by pressing a separate key with the other hand after the flanker task response was made. Both groups were similarly able to signal the occurrence of this perceptual event, with controls reporting it on 81 (21) \% and the mPFC group 81 (8) \% of occurrences. The RT to report the blink was also comparable in the two groups [CTL: 969 (230) ms; dACC: 994 (420) ms; the same patient who could not complete the error reporting task did not perform this task, due to testing session time limitations].

Several studies have indicated that the error-related negativity (ERN) may reflect an "automatic" aspect of error processing, not necessarily related to conscious error detection (Scheffers et al., 1996; Nieuwenhuis et al., 2001). Our finding that mPFC damage disrupts rapid error correction without affecting conscious reporting of errors after they occur is consistent with the findings from these ERP studies, but leaves open whether rapid error prediction is a purely automatic process. The speeded nature of the flanker task makes it difficult to probe pre-response events that might be available to awareness. We therefore undertook a second experiment to examine this question, using a task that allowed measurement of conscious "error likelihood" at the time of the response.

All five patients, and a subset of the control group $(N=10)$ performed an adapted form of the letter two-back task. This task had a go-no go response requirement: a key was to be pressed only if the letter on the screen was a target (i.e., had been presented two trials previously); no response was made if the stimulus was a non-target. We adapted this standard task to tap online monitoring of error likelihood (i.e., confidence), by asking subjects to press one of two keys when a target appeared: one key to indicate that they were certain that the stimulus was a target, and another if they were somewhat certain. No explicit feedback about accuracy was provided. The analysis focused on the level of confidence expressed in this way for correct responses, and for errors of commission.

We have previously reported that patients with dorsal mPFC damage make significantly more errors of commission in the standard version of this task, whether compared with healthy controls, or to patients with damage to other sectors of the frontal lobe (Tsuchida and Fellows, 2008). In that study, the false alarm rate was $>10$-fold higher in those with dorsal mPFC damage compared with healthy control subjects, and voxel-based lesionsymptom mapping established that false alarms were statistically more likely in those with damage to voxels within dorsal ACC compared with damage elsewhere in the frontal lobes. Here, we confirmed that finding at the group level: the mPFC patients made an average of 16.4 (17.1) commission errors, compared with $0.9(1.4)$ in the control group $\left(t_{(13)}=2.46, p<0.05\right)$. There was no significant difference in the number of errors of omission [mPFC: 2.8 (3.8); CTL: 2.6 (3.3)].

The two groups did not differ significantly in their level of confidence for correct go responses; the proportion of correct responses coded as certain was 93 (11) \% in the control group, and $82(16) \%$ in the mPFC group (Fisher's exact test, $p>0.05$ ). On an individual level, only three patients and four controls made at least two errors of commission. In this subset, there was a significant difference in the level of confidence that a response was correct when it was, in fact, an error of commission. Healthy controls indicated that they were only somewhat certain on $70 \%$ of error trials (compared with 15\% of correct trials), whereas those with mPFC damage did so on only $14 \%$ of such trials (compared with $17 \%$ of correct trials) (Fisher's exact test, $p<0.01$ ).

\section{Discussion}

An extensive functional imaging literature argues for a central role for dACC in cognitive control. This role has been variously cast as monitoring response conflict (Botvinick et al., 2001), acting as a response comparator (Ullsperger et al., 2005), detecting errors (Holroyd et al., 2005), predicting error likelihood (Brown and Braver, 2005), engaging attention to overcome prepotent response tendencies (Pardo et al., 1990; Posner and DiGirolamo, 1998; Peterson et al., 1999), or representing action-outcome contingencies (Rushworth et al., 2004; Williams et al., 2004; Rushworth et al., 2007). Several of these accounts have been formalized in computational models (Botvinick et al., 2001; Brown and Braver, 2005, 2008; Holroyd et al., 2005). In this increasingly tightly specified area of investigation, built on extensive evidence from both functional imaging and electrophysiology experiments, the absence of unequivocal loss-of-function evidence in support of any of these hypotheses has been troubling. The present study focused on error-related processes, and provides evidence that dorsal $\mathrm{mPFC}$ is necessary for rapid error prediction.

These findings argue that there are multiple routes to recognizing that an error has occurred. Dorsal mPFC appears to be critical only for the rapid, on-line identification of an error, before (or during) its occurrence. Reporting of an error after it had occurred, over a time course that is consistent with registering the error after the motor response had been produced, remained intact despite dorsal mPFC damage. Early behavioral work established that error correction in choice reaction time tasks occurs more rapidly than the choice reaction time itself, and in some paradigms, is also more rapid than the simple reaction time (Rabbitt, 1966; Angel, 1976). This form of rapid error correction is therefore thought to rely on monitoring of an efference copy of the on-going response. The most intensively studied errorrelated ERP component, the ERN, peaks within $100 \mathrm{~ms}$ of the motor response, and likely arises from dorsal ACC (Dehaene et al., 1994; Herrmann et al., 2004; Vocat et al., 2008). The time course of the ERN also suggests that it relies on monitoring of an efference copy of the motor plan, rather than sensory feedback (Gehring et al., 1993). The present findings support the existence of a distinct error monitoring process relying on efference copy, and provide strong evidence that a region within dorsal $\mathrm{mPFC}$, most likely dorsal ACC, plays a necessary role in this process, either at the point of error detection, or in permitting a rapid corrective response.

The findings from the modified n-back task further suggest that this region is necessary for the conscious evaluation of error likelihood. Thus, mPFC seems to play a critical role in the withintrial modulation of a response by the likelihood that response is an error, a process that has an effect on the readiness to produce the alternate response, and a "conscious" correlate reflected in the overt confidence in the response. These findings together are strongly supported by a functional magnetic resonance imaging 
(fMRI) study which aimed to disentangle conscious, pre-error performance monitoring from the processes involved in error commission (Magno et al., 2006). The task used in that study allowed subjects to avoid errors by rejecting trials they thought they might fail. Dorsal ACC activity was greater for such "reject" trials, compared with trials in which an error was made. This was interpreted as supporting a role for dACC in "on-line adjustment of behavior to prevent errors" (Mango et al., 2006). Our results indicate that dorsal $\mathrm{mPFC}$ plays a necessary role in such a process.

The data presented here do not speak to whether the preresponse processes mediated by dorsal $\mathrm{mPFC}$ are engaged only during error trials, or are present during trials with response competition more generally, although both ERP and fMRI studies suggest the latter (Carter et al., 1998; Botvinick et al., 1999; Van Veen and Carter, 2002). The tendency for those with mPFC damage to have larger flanker effects could be interpreted as reflecting difficulty resolving response conflict even on correct trials. However, in this particular study, this effect should be interpreted with caution, given that task parameters were not identical across patients, or between the patient and control groups. Further, it is striking that although all five patients showed slowing of error correction, the size of the flanker effect was more variable, falling well within the normal range in two of five patients.

In general agreement with the current findings, a previous case report of a patient with a very circumscribed lesion to dACC reported a small, but significant decrement in the rate of error correction in a Stroop variant, with intact post-error slowing (Swick and Turken, 2002). Reaction times for error corrections were not reported, however.

The single dACC-damaged patient reported by Swick and Turken had intact conflict monitoring effects in the Stroop task, despite reduced error correction, further suggesting that error and conflict monitoring do not share a reliance on intact mPFC (Swick and Turken, 2002). At the group level, the current findings, in conjunction with a prior study showing intact conflict monitoring in the Stroop task in a partly overlapping group of patients (Fellows and Farah, 2005) (three patients participated in both studies), also argue for a distinction between error prediction and conflict monitoring, with dorsal mPFC necessary only for the former. However, it may be that the usual behavioral measures of conflict monitoring are more sensitive to postconflict adjustments (which the present data suggest are likely to be intact despite MPFC damage) rather than on the within-trial anticipation of conflict that would be the direct homolog of the error effects we report here.

Interestingly, a recent group study found that sequential (i.e., trial-by-trial) congruency effects in the Simon task were not reliably present after ACC damage (di Pellegrino et al., 2007). These patients also failed to show consistent post-error slowing. In contrast to flanker and Stroop tasks, where cognitive control is likely exerted via selective attention to the relevant stimulus feature (and in turn its associated response), the Simon task more directly taps conflict at the response level (Liu et al., 2004). This distinction, perhaps in addition to differences in precise lesion location (the explanation advanced by di Pellegrino et al.), may be important in reconciling these conflicting findings.

Although several of the patients we studied here had quite extensive mPFC (and in two cases, orbitofrontal cortex) damage, the common area of overlap encompasses dACC, making this region the most likely driver of the slowed error correction that was present in all five patients. Nonetheless, we cannot entirely exclude the possibility that direct damage to, or disruption of connections with nearby regions, including rostral ACC and pre- supplementary motor areas, or indeed disruption of longer range connections, such as via the cingulum bundle or the genu of the corpus callosum, contributes to the effect.

Other recent work has argued for a more nuanced view of dACC's role in response monitoring, one that can account for a putative role for this region in action-outcome learning and related value-related decisions. Our findings indicate that this region is critical for the prediction of response errors, specifically. Further work will be required to determine whether this generalizes to a necessary role in the prediction of the relative value of actions more generally, in contexts where responses have a graded value, rather than being dichotomously "right" or "wrong." Other lines of evidence support this possibility (Gehring and Willoughby, 2002; Rushworth et al., 2004; Quilodran et al., 2008), and one study of the effects of cingulotomy found an increase in errors when response selection was guided by a reduction in reward (Williams et al., 2004). Furthermore, we cannot speak to whether this region is necessary for learning from errors, except to note that one potential index of trial-to-trial adjustment, post-error slowing, was intact despite mPFC damage.

This demonstration of a critical role for dorsal mPFC in only one of several measured error-related processes highlights an important point. Error-related processes are obviously critically important to adaptive behavior, and it seems highly likely that there are multiple neural systems that encode error-related information. Such duplication might compensate for lesion effects, as was demonstrated by the apparent use of "error detection" mechanisms to both report and correct errors after mPFC damage in patients studied here. Deployment of particular error and conflict-related processes appears to differ across individuals, and across task demands (Garavan et al., 2002; Hester et al., 2005; Brown and Braver, 2007). Behavioral measures that focus on the timing of these processes may be helpful in understanding these different mechanisms.

Just as there are likely multiple neural systems involved in error processing, a given neural circuit might be engaged by error prediction in a specific response domain, or serve a more general performance monitoring role across different response types. We recently evaluated memory monitoring in the same five patients studied here in a separate set of experiments. Interestingly, they showed clear deficits in only the prospective, within-trial ability to predict episodic memory performance, as captured by the accuracy of a "feeling-of-knowing" measure (Modirrousta and Fellows, 2008). This supports a more general error likelihood account of dorsal mPFC function, consistent with fMRI findings (De Martino et al., 2006; Magno et al., 2006; Paulus and Frank, 2006; Brown and Braver, 2007), but also helps to pinpoint the timing of the critical contribution of this region to such monitoring as within-trial and before the response.

In summary, damage involving dorsal $\mathrm{mPFC}$ in humans selectively disrupted anticipatory error monitoring, reflected in the speed of error correction and the conscious prediction of errors, while sparing post-response error reporting, and performance adjustment on post-error trials. This result provides important converging evidence for error prediction accounts of the function of this brain region.

\section{References}

Angel RW (1976) Efference copy in the control of movement. Neurology 26:1164-1168.

Baird A, Dewar BK, Critchley H, Gilbert SJ, Dolan RJ, Cipolotti L (2006) Cognitive functioning after medial frontal lobe damage including the anterior cingulate cortex: a preliminary investigation. Brain Cogn 60:166-175. 
Botvinick M, Nystrom LE, Fissell K, Carter CS, Cohen JD (1999) Conflict monitoring versus selection-for-action in anterior cingulate. Nature 402:179-181.

Botvinick MM, Braver TS, Barch DM, Carter CS, Cohen JD (2001) Conflict monitoring and cognitive control. Psychol Rev 108:624-652.

Brown JW, Braver TS (2005) Learned predictions of error likelihood in the anterior cingulate cortex. Science 307:1118-1121.

Brown JW, Braver TS (2007) Risk prediction and aversion by anterior cingulate cortex. Cogn Affect Behav Neurosci 7:266-277.

Brown JW, Braver TS (2008) A computational model of risk, conflict, and individual difference effects in the anterior cingulate cortex. Brain Res 1202:99-108.

Carter CS, van Veen V (2007) Anterior cingulate cortex and conflict detection: an update of theory and data. Cogn Affect Behav Neurosci 7:367-379.

Carter CS, Braver TS, Barch DM, Botvinick MM, Noll D, Cohen JD (1998) Anterior cingulate cortex, error detection, and the online monitoring of performance. Science 280:747-749.

Debener S, Ullsperger M, Siegel M, Fiehler K, von Cramon DY, Engel AK (2005) Trial-by-trial coupling of concurrent electroencephalogram and functional magnetic resonance imaging identifies the dynamics of performance monitoring. J Neurosci 25:11730-11737.

Dehaene S, Posner MI, Tucker DM (1994) Localization of a neural system for error-detection and compensation. Psychol Sci 5:303-305.

De Martino B, Kumaran D, Seymour B, Dolan RJ (2006) Frames, biases, and rational decision-making in the human brain. Science 313:684-687.

di Pellegrino G, Ciaramelli E, Làdavas E (2007) The regulation of cognitive control following rostral anterior cingulate cortex lesion in humans. J Cogn Neurosci 19:275-286.

Eichele T, Debener S, Calhoun VD, Specht K, Engel AK, Hugdahl K, von Cramon DY, Ullsperger M (2008) Prediction of human errors by maladaptive changes in event-related brain networks. Proc Natl Acad Sci U S A 105:6173-6178.

Fellows LK, Farah MJ (2005) Is anterior cingulate cortex necessary for cognitive control? Brain 128:788-796.

Floden D, Stuss DT (2006) Inhibitory control is slowed in patients with right superior medial frontal damage. J Cogn Neurosci 18:1843-1849.

Garavan H, Ross TJ, Murphy K, Roche RA, Stein EA (2002) Dissociable executive functions in the dynamic control of behavior: inhibition, error detection, and correction. Neuroimage 17:1820-1829.

Gehring WJ, Willoughby AR (2002) The medial frontal cortex and the rapid processing of monetary gains and losses. Science 295:2279-2282.

Gehring WJ, Goss B, Coles MGH, Meyer DE, Donchin E (1993) A neural system for error-detection and compensation. Psychol Sci 4:385-390.

Herrmann MJ, Römmler J, Ehlis AC, Heidrich A, Fallgatter AJ (2004) Source localization (LORETA) of the error-related-negativity (ERN/Ne) and positivity (Pe). Brain Res Cogn Brain Res 20:294-299.

Hester R, Foxe JJ, Molholm S, Shpaner M, Garavan H (2005) Neural mechanisms involved in error processing: a comparison of errors made with and without awareness. Neuroimage 27:602-608.

Holroyd CB, Yeung N, Coles MG, Cohen JD (2005) A mechanism for error detection in speeded response time tasks. J Exp Psychol Gen 134:163-191.

Liu X, Banich MT, Jacobson BL, Tanabe JL (2004) Common and distinct neural substrates of attentional control in an integrated Simon and spatial Stroop task as assessed by event-related fMRI. Neuroimage 22:1097-1106.

Magno E, Foxe JJ, Molholm S, Robertson IH, Garavan H (2006) The anterior cingulate and error avoidance. J Neurosci 26:4769-4773.

Mayr U, Awh E, Laurey P (2003) Conflict adaptation effects in the absence of executive control. Nat Neurosci 6:450-452.

Modirrousta M, Fellows LK (2008) Medial prefrontal cortex plays a critical and selective role in 'feeling of knowing' meta-memory judgments. Neuropsychologia 46:2958-2965.
Naccache L, Dehaene S, Cohen L, Habert MO, Guichart-Gomez E, Galanaud D, Willer JC (2005) Effortless control: executive attention and conscious feeling of mental effort are dissociable. Neuropsychologia 43:1318-1328.

Nieuwenhuis S, Ridderinkhof KR, Blom J, Band GP, Kok A (2001) Errorrelated brain potentials are differentially related to awareness of response errors: evidence from an antisaccade task. Psychophysiology 38:752-760.

Pardo JV, Pardo PJ, Janer KW, Raichle ME (1990) The anterior cingulate cortex mediates processing selection in the Stroop attentional conflict paradigm. Proc Natl Acad Sci U S A 87:256-259.

Paulus MP, Frank LR (2006) Anterior cingulate activity modulates nonlinear decision weight function of uncertain prospects. Neuroimage 30:668-677.

Peterson BS, Skudlarski P, Gatenby JC, Zhang H, Anderson AW, Gore JC (1999) An fMRI study of Stroop word-color interference: evidence for cingulate subregions subserving multiple distributed attentional systems. Biol Psychiatry 45:1237-1258.

Picton TW, Stuss DT, Alexander MP, Shallice T, Binns MA, Gillingham S (2007) Effects of focal frontal lesions on response inhibition. Cereb Cortex 17:826-838.

Posner MI, DiGirolamo GJ (1998) Executive attention: conflict, target detection, and cognitive control. In: The attentive brain (Parasuraman R, ed), pp 401-423. Cambridge, MA: MIT.

Quilodran R, Rothé M, Procyk E (2008) Behavioral shifts and action valuation in the anterior cingulate cortex. Neuron 57:314-325.

Rabbitt PM (1966) Error correction time without external error signals. Nature 212:438.

Ridderinkhof KR, Ullsperger M, Crone EA, Nieuwenhuis S (2004) The role of the medial frontal cortex in cognitive control. Science 306:443-447.

Rorden C, Brett M (2000) Stereotaxic display of brain lesions. Behavioral Neurology 12:191-200.

Rushworth MF, Walton ME, Kennerley SW, Bannerman DM (2004) Action sets and decisions in the medial frontal cortex. Trends Cogn Sci 8:410-417.

Rushworth MF, Buckley MJ, Behrens TE, Walton ME, Bannerman DM (2007) Functional organization of the medial frontal cortex. Curr Opin Neurobiol 17:220-227.

Scheffers MK, Coles MG, Bernstein P, Gehring WJ, Donchin E (1996) Event-related brain potentials and error-related processing: an analysis of incorrect responses to go and no-go stimuli. Psychophysiology 33:42-53.

Swick D, Turken AU (2002) Dissociation between conflict detection and error monitoring in the human anterior cingulate cortex. Proc Natl Acad Sci U S A 99:16354-16359.

Tsuchida A, Fellows LK (2008) Lesion evidence that two distinct regions within prefrontal cortex are critical for n-back performance in humans. J Cogn Neurosci, in press.

Ullsperger M, Bylsma LM, Botvinick MM (2005) The conflict adaptation effect: it's not just priming. Cogn Affect Behav Neurosci 5:467-472.

Van Veen V, Carter CS (2002) The timing of action-monitoring processes in the anterior cingulate cortex. J Cogn Neurosci 14:593-602.

Verguts T, Notebaert W (2008) Hebbian learning of cognitive control: dealing with specific and nonspecific adaptation. Psychol Rev 115:518-525.

Vocat R, Pourtois G, Vuilleumier P (2008) Unavoidable errors: a spatiotemporal analysis of time-course and neural sources of evoked potentials associated with error processing in a speeded task. Neuropsychologia 46:2545-2555.

Williams ZM, Bush G, Rauch SL, Cosgrove GR, Eskandar EN (2004) Human anterior cingulate neurons and the integration of monetary reward with motor responses. Nat Neurosci 7:1370-1375.

Yeung N, Botvinick MM, Cohen JD (2004) The neural basis of error detection: conflict monitoring and the error-related negativity. Psychol Rev 111:931-959. 